\title{
Genotypic-specific habitat selection: a new model and its application
}

\author{
Philip W. Hedrick
}

Department of Biology, Pennsylvania State University, University Park, PA 16802, U.S.A.

\begin{abstract}
A new model of genotypic-specific habitat selection is proposed in which habitats are given a biologically meaningful way with differences in the frequency of various niches. Conditions for polymorphism are quite robust even for small viability differences. A method to estimate habitat selection parameters from information on habitat preference is given. Even when habitat preference is not great, it may have a large effect on maintaining polymorphism.
\end{abstract}

\section{INTRODUCTION}

There has been extensive discussion about the impact of habitat selection on the maintenance of genetic variation (e.g., Jones and Probert, 1980; Templeton and Rothman, 1981; Hoekstra et al., 1985; Rausher 1985; Garcia-Dorado, 1986, 1987; Hedrick 1986; Jaenike and Holt, 1990; Hedrick, 1990). When habitat selection is genotypic-specific, it generally appears to enhance greatly the potential for such maintenance in heterogeneous environments (e.g., Hedrick, 1986). However, most genotypic-specific habitat selection models assume that habitat choice for a given genotype is constant and does not depend on the frequency of the appropriate niche. Here I compare the constant genotypic-specific habitat selection model to two models that take into account habitat frequency and show how the habitat selection parameters can be estimated from habitat preference data and applied to such a model.

\section{MODELS OF HABITAT SELECTION}

The simplest model of genotypic-specific habitat selection is that each genotype at a biallelic locus selects between two different niches. General habitat selection values and relative fitness values for this case are given in table 1 . Templeton and Rothman (1981) who introduced this model, assumed that habitat selection was constant for a given genotype and was independent of the
Table 1 Habitat selection and viability selection when there are two niches

\begin{tabular}{lllll}
\hline & & \multicolumn{3}{c}{ Genotype } \\
\cline { 3 - 5 } & Niche & $\mathrm{A}_{1} \mathrm{~A}_{1}$ & $\mathrm{~A}_{1} \mathrm{~A}_{2}$ & $\mathrm{~A}_{2} \mathrm{~A}_{2}$ \\
\hline Habitat & 1 & $h_{11.1}$ & $h_{12.1}$ & $h_{22.1}$ \\
selection & 2 & $1-h_{11.1}$ & $1-h_{12.1}$ & $1-h_{22.1}$ \\
Viability & 1 & $w_{11.1}$ & $w_{12.1}$ & $w_{22.1}$ \\
selection & 2 & $w_{11.2}$ & $w_{12.2}$ & $w_{22.2}$ \\
\hline
\end{tabular}

frequency of the different environments. However, when a niche becomes quite rare, it seem biologically unrealistic to assume that the same proportion of animals will select it as when it is common. Actually, Garcia-Dorado (1987) assumed that habitat selection is a function of the habitat frequency (as first proposed by Maynard Smith (1966)). Even this model does not seem completely appropriate, as I will show below.

For simplicity in comparing these models, let us assume that heterozygotes are intermediate, that the preference of $A_{1} A_{1}$ for niche 1 is equivalent to the preference of $\mathrm{A}_{2} \mathrm{~A}_{2}$ for niche 2, and that the frequencies of niches 1 and 2 are $c_{1}$ and $c_{2}$, respectively (see table 2). To illustrate the difference between them assume that $c_{1}$ is near 0 , i.e., niche 1 is extremely rare. Because the habitat selection model of Templeton and Rothman is independent of $c_{1}$, habitat selection does not change in their model as $c_{1}$ changes, leading to the situation in which a proportion $h$ of $\mathrm{A}_{1} \mathrm{~A}_{1}$ selects niche 1 even though this niche is very rare. 
Table 2 Three different models of genotypic-specific habitat selection

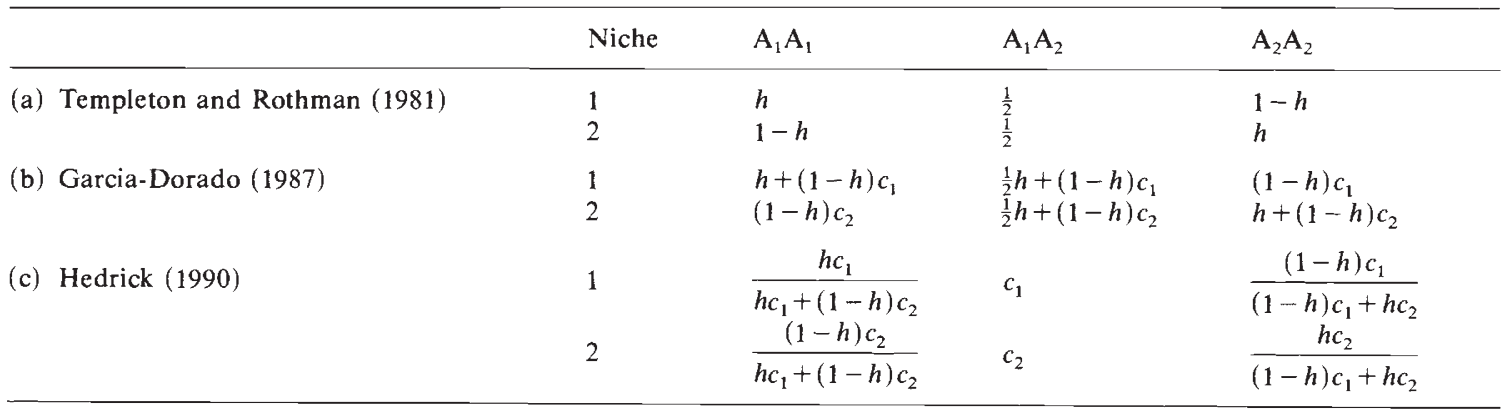

Garcia-Dorado's (1987) genotypic-specific habitat selection model assumes that a fraction $h$ of homozygotes chooses the niche to which they are optimally adapted and a fraction $1-h$ settles at random, giving the habitat selection values in table $2(\mathrm{~b})$. If we again assume $c_{1}$ is near 0 , then the habitat selection values in this model approach $h, \frac{1}{2} h$, and 0 in niche 1 and $1-h, 1-\frac{1}{2} h$, and 1 in niche 2 for genotypes $A_{1} A_{1}, A_{1} A_{2}$ and $A_{2} A_{2}$, respectively. Once again it does not seem biologically realistic that a proportion $h$ of $A_{1} A_{1}$ selects the extremely rare niche 1 .

A straightforward way to circumvent these problems is to use a model based on the proportions of the two niches and habitat preference as given in table 2(c) (Hedrick, 1990). Here the habitat selection values depend on the frequency of encounter of the different niches and on innate preferences in a more realistic manner than that suggested by Garcia-Dorado. For example, if $c_{1}$ is near 0 then the habitat selection values in niche 1 are also near 0 for all genotypes.

\section{MAINTENANCE OF POLYMORPHISM}

These three models of habitat selection differ in their ability to maintain variation at a locus undergoing viability selection. Using the model given in table 1, Templeton and Rothman (1981) and Garcia-Dorado (1987) show that the conditions for a stable polymorphism are

$$
\sum_{i} c_{i} \frac{h_{12 . i} w_{12 . i}}{h_{11 . i} w_{11 . i}}>1
$$

and

$$
\sum_{i} c_{i} \frac{h_{12 . i} w_{12 . i}}{h_{22 . i} w_{22 . i}}>1 .
$$

These conditions can be written in terms of the harmonic mean fitnesses of the three genotypes weighted by the habitat selection values. The weighted harmonic mean fitness of heterozygotes must be greater than that of the two homozygotes for a stable polymorphism. When there are no differences in viability and only two niches, then the conditions for a stable polymorphism are reduced to $h_{11.1}>c_{1}>h_{22.1}$ (see also Rausher, 1984).

These conditions for a polymorphism can be used to compare the three habitat selection models. Figure 1 gives the limits of $c_{1}$ between the symmetrical lines for the full range of $s$ when there is $\frac{1}{4}$ of the maximum habitat selection $(h=0.625$ for the models of Templeton and Rothman and of

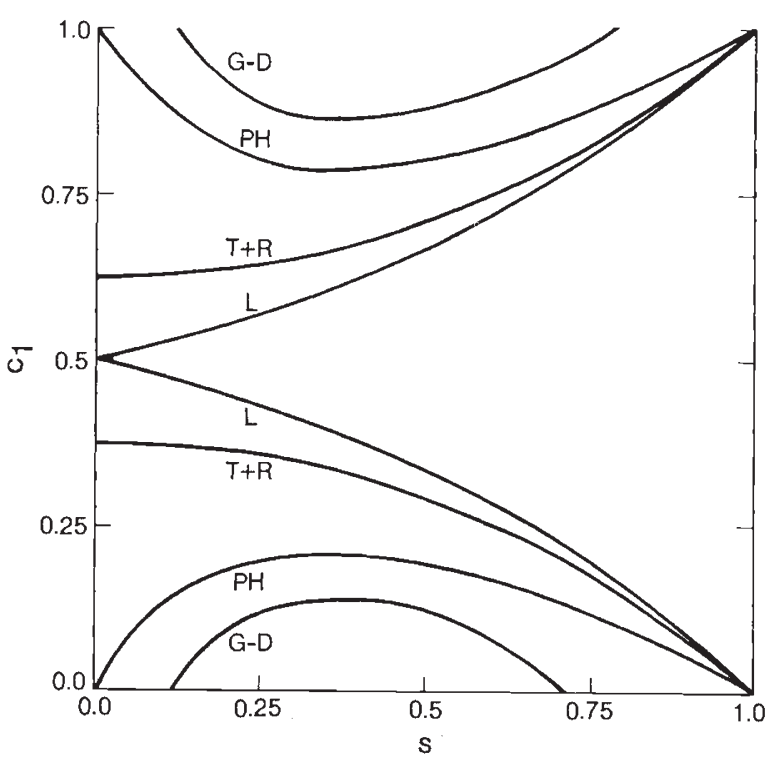

Figure 1 Comparison of the regions of stability (between pairs of curved lines) for the Levene model (L) and the three models of habitat selection ( $h=0.625$ for the models of Templeton and Rothman $(\mathrm{T}+\mathrm{R})$ and Hedrick $(\mathrm{PH}), h=$ 0.25 for the model of Garcia-Dorado (G-D)) where $c_{1}$ is the proportion of niche 1 and $s$ is selective difference between the two homozygotes. 
Hedrick and $h=0.25$ for the model of GarciaDorado). It is assumed here that the relative fitnesses of genotypes $A_{1} A_{1}, A_{1} A_{2}$, and $A_{2} A_{2}$ are $1,1-\frac{1}{2} s$, and $1-s$ in niche 1 and the fitness of the two homozygotes are reversed in niche 2 . For comparison, the limits under the Levene (1953) model, which has no habitat selection, are also given. All of these genotypic-specific habitat selection models broaden the conditions for a polymorphism compared to the conditions under the Levene model, with greatest effect under the GarciaDorada model and the least under Templeton and Rothman. When there is no viability selection ( $s=$ 0 ), there is a stable polymorphism for all proportions of the two environments for the GarciaDorado model and the Hedrick model, while for the Templeton and Rothman model, the limits are between $c_{1}=h$ and $1-h$. Most important, all of the models significantly broaden the conditons when $s$ is low (see Hedrick, 1990 for further discussion).

\section{ESTIMATION OF HABITAT SELECTION FROM HABITAT PREFERENCE DATE}

There have been several tests of habitat preference of among strains of insects, particularly Drosophila (e.g., Hoffmann, 1985; Jaenike, 1985). The information from these studies can be used to estimate the habitat selection parameters in the model given above. To do so, data from preference experiments can be organized as in table 3 (a) (usually only two groups have been used in these tests). For example, using the habitat selection model in table 2(c), we can assume that the proportion of genotype (or group) 1 found in niche 1 is

$$
h_{1.1}=\frac{h_{1} c_{1}}{h_{1} c_{1}+\left(1-h_{1}\right)\left(1-c_{1}\right)}
$$

where $h_{1}$ corresponds to $h$ above for group or genotype 1 (we are assuming $h$ may vary for different groups or genotypes here). The propor-

Table 3 Estimation of habitat selection from habitat preference data. The numbers in (b) to (d) are the proportions and the $c_{i}$ value calculated as given in (a).

(a)

\begin{tabular}{|c|c|c|c|c|c|c|}
\hline & & \multicolumn{4}{|c|}{ Genotype or group } & \multirow[b]{3}{*}{$c_{i}$} \\
\hline & & \multicolumn{2}{|c|}{1} & \multicolumn{2}{|c|}{1} & \\
\hline & & Number & Proportion & Number & Proportion & \\
\hline \multirow[t]{2}{*}{ Niche } & 1 & A & $h_{1.1}=\frac{A}{A+C}$ & $B$ & $1-h_{2.2}=\frac{B}{B+D}$ & $c_{1}=\frac{1}{2}\left(h_{1.1}+1-h_{2.2}\right)$ \\
\hline & 2 & $C$ & $1-h_{1.1}=\frac{C}{A+C}$ & $D$ & $h_{2.2}=\frac{D}{B+D}$ & $c_{2}=\frac{1}{2}\left(1-h_{1.1}+h_{2.2}\right)$ \\
\hline
\end{tabular}

(b) Jaenike (1985)

\begin{tabular}{llll}
\hline & $\mathrm{S} 64$ & $\mathrm{~S} 74$ & $c_{i}$ \\
\hline Mushroom & 0.605 & 0.158 & 0.382 \\
Tomato & 0.395 & 0.842 & 0.618 \\
\hline
\end{tabular}

(c) Hoffmann and O'Donnell (1990)

\begin{tabular}{llll}
\hline & Lemon & Orange & $c_{i}$ \\
\hline Lemon & 0.956 & 0.914 & 0.935 \\
Orange & 0.044 & 0.086 & 0.065 \\
\hline
\end{tabular}

(d) Hey and Houle (1986)

\begin{tabular}{llll}
\hline & Hemlock & Deciduous & $c_{i}$ \\
\hline Hemlock & 0.707 & 0.632 & 0.670 \\
Deciduous & 0.293 & 0.368 & 0.330 \\
\hline
\end{tabular}


tion of niche 1 can be calculated as

$$
c_{1}=\frac{1}{2}\left(h_{1.1}+1-h_{2.2}\right) \text {. }
$$

Although in all the experiments discussed below, equal numbers of the two groups were released, this method of calculating $c_{1}$ avoids the bias that may occur if unequal numbers of the two groups are caught. Solving expression (2) for $h_{1}$ gives

$$
h_{1}=\frac{h_{1.1}\left(1-c_{1}\right)}{c_{1}+h_{1.1}\left(1-2 c_{1}\right)}
$$

and likewise solving the complementary expression for $h_{2}$ gives

$$
h_{2}=\frac{h_{2.2} c_{2}}{c_{2}-h_{2.2}\left(1-2 c_{1}\right)}
$$

The estimation of the habitat selection parameters using this approach can be illustrated with three studies that show statistically significant habitat preference. Jaenike (1985) compared the attractiveness of mushrooms and tomatoes in two strains of Drosophila tripuncata, Hoffmann and O'Donnell (1990) compared the attractiveness of lemons and oranges for groups of $D$. melanogaster collected on lemons and oranges, and Hey and Houle (1988) compared the attractiveness of hemlock and deciduous habitat for groups of $D$. affinis males collected in each of them.

Jaenike's (table 3(b)), give $h_{1}=0.712$ and $h_{2}=$ 0.768 indicating that strain $\mathrm{S} 64$ strongly prefers mushrooms and strain S74 strongly prefers tomatoes. These strong and symmetrical preference values were obvious from the original data and from other experiments. For Hoffmann and O'Donnell (table 3(c)), $h_{1}=0.603$ and $h_{2}=0.648$, illustrating that the preferences are symmetrical even though the two niches have greatly different overall attractiveness. In this case, the measure of habitat preference introduced by Turelli et al. (1984), $\Delta=h_{1.1}-\left(1-h_{2.2}\right)$, is $0 \cdot 04$, a small, but statistically significant, value (Hoffmann and O'Donnell, 1990). Finally, in the example from Hey and Houle (table 2(d)), $\Delta=0.075$, again a statistically significant value. However, in this case the habitat selection values are quite small, $h_{1}=$ 0.543 and $h_{2}=0.542$, values that are much closer to 0.5 than those found for Jaenike or for Hoffmann and O'Donnell.

\section{DISCUSSION}

Although habitat selection may broaden the conditions for a stable polymorphism, only when there is genotypic-specific habitat selection are the conditions greatly expanded. Several models of genotypic-specific habitat selection have been suggested (e.g., Templeton and Rothman, 1981; Garcia-Dorado, 1987) although these models do not adequately account for differences in niche frequency. As a result, I have proposed another model that includes niche frequency into the habitat selection values in a biologically meaningful manner.

A number of studies have estimated habitat preferences in Drosophila (see reviews in Hedrick, 1986; Hoffmann and O'Donnell, 1990). Here I have shown how these habitat preference data can be used to give a measure of the habitat selection parameter(s) in the model I proposed above and applied this approach to three data sets. In the three examples given, the habitat selection value for the Jaenike data are quite large (larger than the theoretical values used in fig. 1); the values for the Hoffmann and O'Donnell data are of the same magnitude, and those of Hey and Houle are smaller. In other words, even though the habitat selection parameters estimated from the Hoffmann and O'Donnell data to result in fairly subtle habitat preference, they could exert a significant influence on the maintenance of genetic polymorphism.

The habitat preferences observed here and in other studies (e.g., Hedrick, 1990; and Hoffmann and O'Donnell, 1990) are likely to be polygenically based (although the attraction to mushrooms or tomatoes in D. tripunctata (Jaenike, 1985) appears to be under simple genetic control). However, unlike many other models of variable environments (e.g., Hedrick et al., 1976; Maynard Smith and Hoekstra, 1980; Hoekstra et al., 1985) genotypic-specific habitat selection models are robust even for small selective differences. As a result, it is possible that subtle genotypic-specific habitat preference, even for polygenic traits, may be of major importance in the maintenance of genetic polymorphism.

Acknowledgement I appreciate the comments of Stuart Barker, Ary Hoffmann, and John Jaenike on various aspects of this research. This study was initiated while I held a Visiting Research Fellowship at the University of New England, Australia.

\section{REFERENCES}

GARCIA-DORADO, A. 1986. The effect of niche preference on polymorphism protection in a heterogeneous environment. Evolution, 40, 936-945. 
GARCIA-DORADO, A. 1987. Polymorphism from environmental heterogeneity: some features of genetically induced niche preference. Theoret. Pop. Biol., 32, 66-75.

HEDRICK, P. W., GINEVAN, M. E. AND EWING, E. P. 1976. Genetic polymorphism in heterogeneous environments. Ann. Rev. Syst. Ecol., 17, 535-566.

HEDRICK, P. W. 1986. Genetic polymorphism in heterogeneous environments: A decade later. Ann. Rev. Ecol. Syst., 17, $535-566$.

HEDRICK, P. W. 1990. Theoretical analysis of habitat selection and the maintenance of genetic variation. In Barker, J. S. F., Starmer, W. T. and McIntyre, R. (eds) Ecological and Evolutionary Genetics of Drosophila, Plenum, New York.

HEY, J., AND HOULE, D. 1987. Habitat choice in the Drosophila affinis subgroup. Heredity, 58, 463-471.

hOEKSTRA, R. F., BIJLSMA, R. AND DOLMAN, A. J. 1985. Polymorphism from environmental heterogeneity: Models are only robust if the heterogygote is close in fitness to the favored homozygote in each environment. Genet. Res., 45 , 299-319.

HOFFMANN, A. A. 1985. Effects of experience on oviposition and attraction in Drosophila; comparing apples and oranges. Amer. Natur., 126, 41-51.

HOFFMANN, A. A. AND O'DONNELL, S. 1990. Heritable variation in resource use in Drosophila in the field. In Barker, J. S. F., Starmer, W. T. and McIntyre, R. (eds). Ecological and Evolutionary Genetics of Drosophila, Plenum, New York.
JAENIKE, J. 1985. Genetic and environmental determinants of food preference in Drosophila tripunctata. Evolution, 39, 362-369.

JAENIKE, J. AND HOLT, R. D. 1990. Genetic variation for habitat preference: evidence and explanations. Amer. Natur., (In press).

JONES, J. S. AND PROBERT, R. F. 1980. Habitat selection maintains a deleterious allele in a heterogeneous environment. Nature 287, 632-633.

LEVENE, H. 1953. Genetic equilibrium when more than one ecological niche is available. Amer. Natur., 87, 331-333.

MAYNARD SMITH, J. 1966. Sympatric speciation. Amer. Natur., 107, 171-198.

MAYNARD SMITH, J. AND HOEKSTRA, R. F. 1980. Polymorphism in a varied environment: How robust are the models? Genet. Res., 35, 45-57.

RAUSHER, M. D. 1984. The evolution of habitat preference in subdivided populations. Evolution, 38, 596-608.

TEMPLETON, A. R. AND ROTHMAN, E. D. 1981. Evolution in fine-grained environments. II Habitat selection is a homeostatic mechanism. Theoret. Pop. Biol., 19, 326-340.

TURELli, M., COYNE, J. A. AND PROUT, T. 1984. Resource choice in orchard populations of Drosophila. Biol. J. Linn. Soc., 22, 95-106. 